Dialectologia. Special issue, IX (2021), 285-310.

ISSN: 2013-2247

Received 19 April 2021.

Accepted 30 April 2021.

\title{
CAMBIO LINGÜÍSTICO Y VARIACIÓN EN EL GALLEGO RURAL DEL SIGLO XX: LOS PERFECTOS VERBALES ${ }^{1}$
}

\author{
Clara LAGO CAAMAÑo \& Xulio SOUSA \\ Instituto da Lingua Galega, Universidade de Santiago de Compostela, Spain * \\ claralago.caamano@usc.gal \&xulio.sousa@usc.gal
}

Resumen

El empleo de los atlas lingüísticos como fuente de documentación para el estudio del cambio lingüístico no tiene todavía mucha tradición en el ámbito de los estudios románicos. A pesar de la existencia de materiales recogidos para proyectos de geografía lingüística en distintos dominios y épocas, son escasos los trabajos que aprovechan estos materiales para conocer las modificaciones que se produjeron en las estructuras lingüísticas después de los inicios del siglo XX. La información geolingüística permite conocer la repercusión que las importantes transformaciones sociales, económicas y culturales tuvieron sobre las variedades rurales de las lenguas europeas. Este trabajo tiene como propósito mostrar los resultados del estudio de las mudanzas lingüísticas en el gallego rural a lo largo del siglo pasado a partir de la información albergada en dos atlas lingüísticos desarrollados en distintos momentos ( $A L P I$ y $A L G a)$. Las variables analizadas corresponden a la morfología de los tiempos verbales de perfecto y las variantes estudiadas fueron registradas en localidades del dominio lingüístico gallego. Los resultados del trabajo muestran la utilidad de los materiales geolingüísticos para conocer los procesos de cambio producidos en épocas recientes.

\footnotetext{
${ }^{1}$ Este trabajo se ha realizado en el marco del proyecto de investigación "Variación y cambio en los dominios gallego y portugués en el siglo XX: análisis del habla rural a partir de materiales geolingüísticos", financiado por el Ministerio de Ciencia, Innovación y Universidades (PGC2018-095077B-C44; MCIU/AEI/FEDER, UE).

* Instituto da Lingua Galega, Praza da Universidade 4, 15782 Santiago de Compostela.
} 


\title{
Palabras clave
}

dialectología, cambio lingüístico, gallego, morfología, tiempos de perfecto

\section{LANGUAGE CHANGE AND VARIATION IN THE GALICIAN SPOKEN IN RURAL AREAS IN THE 20th CENTURY: PERFECT TENSES}

\begin{abstract}
The use of linguistic atlases to document and study language change is still a new practice in Romance language studies. Despite the existence of material gathered in the framework of language geography projects in different domains and timeframes, few studies have used this material to analyse changes that occurred in language structures since the beginning of the 20th century. Geolinguistic information allows studying the repercussions that significant social, economic, and cultural transformations have had on rural varieties of European languages. This paper aims to show the results of research on language change in the Galician spoken in rural areas in the past century parting from information in two language atlases compiled in two different moments ( $A L P I$ and $A L G a)$. We analyse perfect tense variables documented in places from the Galician language domain. The results prove the importance of geolinguistic material for understanding recent language change.
\end{abstract}

\section{Keywords}

dialectology, language change, Galician, morphology, perfect tenses

\section{Introducción}

El estudio del cambio lingüístico en tiempo real acostumbra a fundamentarse en la comparación de documentación escrita de distintos momentos del pasado con producciones orales y escritas del presente. Hasta la extensión del uso de grabadoras de sonido, la transcripción fonética era la mejor forma que tenían los estudiosos de capturar con fidelidad la lengua oral. Las investigaciones de dialectología sirvieron como terreno de pruebas para muchos de los sistemas de transcripción que se fueron proponiendo a partir del siglo XIX (Kemp 2006) y los proyectos de atlas lingüísticos que se desarrollaron en Europa, fundamentalmente a partir del primer cuarto del siglo pasado, fueron aprovechando, adaptando y perfeccionando estos sistemas de notación. Los atlas lingüísticos acabaron convirtiéndose en los testimonios más fiables 
de la lengua hablada utilizados por los lingüistas (Cukor-Avila \& Bailey 2013: 254-258). Los primeros atlas se concibieron como obras de documentación de las hablas rurales en un momento de transformación de las sociedades tradicionales y por lo tanto como fuente documental del estado pasado de la lengua. El Atlas Linguistique de la France $(A L F)$ de Gilliéron, prototipo de la primera generación de proyectos de geografía lingüística, tuvo como principal propósito comprobar empíricamente la validez y constancia de las leyes fonéticas planteadas por los comparatistas (Dalbera 2007). Los resultados de la investigación del $A L F$ y de otros trabajos de dialectología acabaron refutando la validez de las leyes fonéticas y en consecuencia los objetivos de las investigaciones dialectales tuvieron que ser revisados. Los atlas lingüísticos posteriores pasaron a concebirse como proyectos de documentación de la situación lingüística de la lengua en un momento concreto y tuvieron como finalidad conocer y describir la distribución geográfica de los hechos lingüísticos (González 1991). No se abandonaba de todo el propósito inicial de comparación, pero cobraba mayor relevancia la búsqueda de relaciones entre los hechos lingüísticos y factores externos de distinto tipo (históricos, sociales, geográficos, demográficos, económicos, religiosos, etc.; Cugno \& Massobrio 2014).

El proyecto del Atlas Lingüístico de la Península Ibérica $(A L P I)$ se emprende a inicios del siglo pasado en el marco de la dialectología tradicional. Menéndez Pidal, promotor de la empresa, señala la función que según él debían cumplir los trabajos de dialectología en el ámbito del español: "conocer las múltiples variedades dialectales que aún subsisten en España, y deslindarlas en el mapa para tener una idea del habla viviente que late debajo de la uniformidad literaria" (Cortés \& García 2009: 51, carta a Unamuno de 1910). ${ }^{2}$ El ilustre filólogo era consciente de que los materiales dialectales podían ser aprovechados también para investigar las transformaciones que se producían en la lengua con el paso del tiempo. En un pasaje de Orígenes del español Pidal se adelanta al debate metodológico sobre las distintas formas de abordar el

\footnotetext{
${ }^{2}$ Tanto Gilliéron como Menéndez Pidal en sus estudios sobre los materiales dialectales buscaban reconstruir un estado anterior de la lengua, no a partir de la comparación, sino basándose en la distribución geográfica de las variantes (vid. Vidos 1973: 45).
} 
estudio del cambio lingüístico que se produciría cinco décadas después (tiempo real vs. tiempo aparente; vid. Cukor-Avila \& Bailey 2013).

Los observadores de la lengua viva de una localidad comprenden que un cambio fonético necesita para desarrollarse el concurso de varias generaciones; pero su observación, limitada a un momento, estudia esas generaciones convivientes entre sí: la vieja, la madura, la joven; y ese espacio de tiempo es insignificante para el desarrollo de un cambio; la dialectología para dar sus resultados completos necesitará observaciones reiteradas sobre una misma localidad hechas con bastantes años de intervalo. Se ha buscado el principio de la evolución del lenguaje en cada cambio de generación; pero las generaciones no cambian cada veinte o cada treinta años, sino que cada día nacen y se renuevan imperceptiblemente. Cualquier cambio en la actividad colectiva tradicional, lo mismo respecto al lenguaje que a la canción popular, que a la costumbre jurídica, etc., se funda en el hecho de que muchas generaciones consecutivas participan de una misma idea innovadora y la van realizando persistentemente, a pesar de pequeñas variantes en el modo de concebirla; constituyen una tradición nueva, en pugna con otra tradición más antigua (Menéndez Pidal, 1926: 562)

Los datos registrados en los trabajos de dialectología son una imagen de la lengua en un momento preciso de su evolución y como los anillos de un árbol servirán para revelar los cambios que se van produciendo de generación en generación.

Casi cincuenta años después de iniciado el proyecto del $A L P I$, en el último tercio del siglo XX, se emprende el proyecto del Atlas Lingüístico Galego ( $A L G a)$, una investigación dialectal que sigue el modelo de los atlas tradicionales de pequeño dominio, tanto en sus propósitos como en su metodología. Los responsables de la investigación, dirigida por Constantino García y Antón Santamarina, declaran que los objetivos perseguidos en la empresa son múltiples: i) estudiar la variación diatópica en los distintos niveles de análisis (fonética, morfología, sintaxis y léxico); ii) elaborar un diccionario del habla viva; iii) testimoniar la influencia de los factores sociales en la variación (edad, clase, ocupación, formación, etc.); y iv) identificar el sentido de los cambios lingüísticos (García et al. 1977: 9-10). A pesar de estas ambiciosas pretensiones y de que los métodos de la dialectología tradicional ya se habían visto afectados por la revolución sociolingüística, el $A L G a$ acaba siendo un atlas lingüístico 
tradicional que pretende profundizar en conocimiento del gallego rural de mediados de la década de los años setenta. Para cumplir el último de los objetivos señalados por los promotores del proyecto sería necesario otro término de comparación, que los investigadores no aclaran si se encontraba en el pasado o si sería necesario esperar al avance de la lingüística gallega.

El $A L P I$, por su propósito, objetivos y método, cumple los requisitos para ser utilizado como fuente documental de una variedad lingüística confrontable con la registrada en el ALGa. La variedad documentada en estas dos obras es la examinada en los estudios de dialectología tradicional y la propia de los hablantes que los investigadores de esta corriente consideraban como auténticos. Salvando las diferencias temporales, para las dos obras son aplicables las palabras con que Navarro Tomás describe el propósito del ALPI: "recoger el material necesario para ofrecer una representación de la lengua popular hablada en pueblos menores y antiguos por personas iletradas o de escasa cultura, entre los cuarenta y los setenta años de edad" (Navarro Tomás 1975: 9).

Este artículo presenta los resultados del análisis de un conjunto de variables de morfología verbal presentes en los materiales de los dos atlas lingüísticos realizados en dominio gallego: el Atlas Lingüístico de la Península Ibérica $(A L P I)$ y el Atlas Lingüístico Galego. Los datos analizados corresponden a 61 localidades investigadas para estas obras en dos momentos del siglo pasado. El objetivo es indagar si estos materiales, registrados con casi medio siglo de distancia, pueden contribuir al conocimiento de los cambios lingüísticos que se produjeron en las hablas rurales gallegas durante este período. En la siguiente sección se presentan las características fundamentales de los dos proyectos y se justifica su empleo para el estudio del cambio en tiempo real. A continuación, se describen y analizan el conjunto de variables de morfología verbal de formas de perfecto mostrando las similitudes y diferencias que se aprecian en los datos de las dos fuentes. El resultado de este análisis es utilizado para presentar una reflexión final sobre el valor de los atlas lingüísticos como fuente de documentación de 
estudios de cambio lingüístico y de forma particular para conocer los cambios en el gallego rural durante el siglo XX.

\section{El gallego rural del siglo XX en los atlas lingüísticos}

La curiosidad por el estudio de las variedades lingüísticas del gallego surge en el ambiente ilustrado del siglo XVIII al mismo tiempo que el interés por los dialectos en el resto de los dominios peninsulares (Sousa en prensa). El ilustrado benedictino Martín Sarmiento es citado como el precursor de la dialectología en la península ibérica (Gimeno Menéndez 1990), a pesar de que en sus obras las observaciones sobre información léxica y gramatical aparecen desperdigadas en distintos escritos y acompañando siempre a notas sobre botánica, folclore, historia o geografía. En la península el estudio propiamente científico de las variedades se inició en los comienzos del siglo XX con los escritos de Menéndez Pidal sobre áreas limítrofes entre dominios lingüísticos y los trabajos de Antoni Griera para la elaboración del Atlas Lingüístic de Catalunya. En el ámbito del gallego, un grupo de investigadores formados en la escuela de Hamburgo emprendieron en las primeras décadas de siglo varias investigaciones de campo de carácter etnolingüístico sobre zonas de las provincias de Lugo, Ourense y A Coruña. Sin embargo, no fue hasta los años precedentes al inicio de la guerra civil cuando el gallego aparece como objeto de estudio de un proyecto geolingüístico que pretendía estudiar "toda la unidad lingüística románica de la Península" (Navarro 1975: 9). El resultado del trabajo realizado por los investigadores de este proyecto ofrece una imagen detallada de las hablas rurales de más de medio centenar de localidades comprendidas en la parte gallega del área denominada como "Zona gallego-portuguesa" en el ALPI.

En las décadas posteriores los estudios sobre el gallego hablado son escasos. Las contribuciones más destacadas son las de Zamora Vicente, publicadas a mediados de siglo, que ofrecen una imagen bastante completa de la variación dialectal ligada a un conjunto de fenómenos fonéticos y morfológicos (Fernández Rei 2007). En la década 
de los años setenta, cuarenta años después de realizadas las encuestas para el ALPI en Galicia, se emprende un nuevo proyecto de atlas lingüístico (Atlas Lingüístico Galego, $A L G a)$, esta vez centrado en el dominio gallego y siguiendo el modelo de los atlas regionales realizados por Manuel Alvar (García Mouton 2014).

Los dos proyectos geolingüísticos fueron concebidos en el marco de la dialectología tradicional y como consecuencia comparten varias características relacionadas con el propósito, el objeto estudiado y la metodología empleada (Tabla 1). Las diferencias, evidentes y significativas, no impiden que los datos obtenidos en las dos investigaciones puedan ser utilizados para conocer y comparar el estado de las hablas rurales en dos momentos del siglo XX. El $A L G a$, concebido como un atlas de pequeño dominio, tiene una red de puntos más densa (167 puntos en Galicia y provincias limítrofes de habla gallega) y entre sus objetivos figuraba la intención de recopilar el mayor número de variantes para cada una de las variables estudiadas. El $A L P I$, en cambio, es un proyecto de amplio dominio y su red de localidades es menos densa (53 puntos en Galicia y 8 fuera del territorio gallego). Además, el cuestionario del ALGa contaba con más del doble de preguntas que los dos cuadernos del ALPI. Con todo, es evidente que el tipo de variedad investigada en los dos proyectos es muy similar y responde al perfil de lo que la dialectología presociolingüística identificaba como propia del hablante genuino (Britain 2014): personas de edad avanzada, con poca movilidad fuera de su localidad, residentes en zonas rurales $y$, fundamentalmente, de sexo masculino.

La información contenida en los cuadernos de ambas obras es un testimonio insubstituible de las variedades lingüísticas empleadas en un entorno social y cultural que sufrió muchas transformaciones a lo largo del siglo pasado. 


\begin{tabular}{lll} 
& ALPI & ALGa \\
\hline Edad media de los informantes & 59 & 47 \\
Número de preguntas del cuestionario & 1244 & 2700 \\
Número de localidades investigadas & 61 & 167 \\
Fecha de realización de las entrevistas & $1934-1935$ & $1974-1977$
\end{tabular}

Tabla 1. Comparación entre las características de los dos proyectos.

De las diferencias que existen entre las dos fuentes escogidas, las más relevantes para este trabajo son las que tienen que ver con la metodología de encuesta utilizada para registrar las variables de morfología verbal. En el $A L G a$, como señalan los investigadores (García et al. 1977), se utilizó un método encuesta indirecto. Este método, junto con el afán por recoger toda la información posible y los distintos materiales considerados como fuentes, hace que sea habitual que para una única variable se consignen varias respuestas. En la introducción del volumen publicado del $A L P I$ se declara que se procuró utilizar un método indirecto para obtener la información (Navarro Tomás et al. 1962: 10). Al analizar las respuestas recogidas para las preguntas del apartado de morfosintaxis se comprueba que posiblemente los investigadores utilizaron con frecuencia el método directo. No es arriesgado suponer que como estímulo se debió utilizar el enunciado de la pregunta en español o una forma convencionalizada de la variedad local. En las cuestiones de las secciones de morfología y sintaxis se buscaba información a partir de estímulos oracionales muy concretos, y posiblemente este método condicionó las respuestas obtenidas.

A pesar de las diferencias, los dos proyectos muestran suficientes características comunes para justificar que sean utilizados como fuentes para el estudio del cambio lingüístico en tiempo real. Los aspectos que comparten ofrecen suficientes garantías para la comparación, siempre que se obre con cautela al extraer conclusiones. Los resultados deben ser interpretados sin olvidar las diferencias metodológicas y considerando el perfil de los hablantes seleccionados (Sousa \& Rodríguez 2017). 


\section{Datos y variables analizadas}

La información analizada en este trabajo está tomada del cuestionario del primer cuaderno del ALPI y del volumen de morfología verbal del ALGa (Fernández Rei 1990a). Alrededor de 40 preguntas del cuestionario I del ALPI abordan variables que incluyen datos para algún verbo en pretérito perfecto. El primer tomo del volumen del ALGa se dedica a los verbos regulares y el segundo a los irregulares. Los mapas se ordenan en los volúmenes teniendo en cuenta los tiempos y personas de los paradigmas (Fernández Rei 1993). Es necesario señalar que para la elaboración de los mapas del volumen I del $A L G a$, además de la información obtenida en las entrevistas, se utilizó un cuestionario complementario específico de morfología verbal y también la información extraída de grabaciones del Arquivo Dialectolóxico del ILG, recogidas en distintos momentos entre 1977 y 1984 (Fernández Rei 1993). En consecuencia, se incrementó el número de localidades con puntos secundarios y también de respuestas distintas asociadas a un mismo punto. En este trabajo se consideran únicamente las variantes registradas en los puntos principales del $A L G a$.

Para poder realizar la comparación se buscaron las mismas formas verbales en las preguntas de las dos obras o bien aquellas que muestran en las repuestas un padrón de conjugación equivalente. La variables seleccionadas y analizadas son 7: cuatro que afectan a verbos regulares y otras tres relacionadas con el paradigma de tres verbos irregulares (Tabla 2).

Con respecto a la selección de puntos, se partió de las 61 localidades del dominio gallego estudiadas en el $A L P I$ y se escogieron los puntos del $A L G a$ próximos, de acuerdo con el procedimiento propuesto en trabajos precedentes (Sousa 2010, 2012, Sousa \& Rodríguez 2017, Lago 2018; véase la relación de puntos y el mapa en los anexos 1 y 2 ). 


\begin{tabular}{lll} 
VARIABLE & ALPI (№ DE PREGUNTA) & ALGA (№ MAPA) \\
\hline $\begin{array}{l}\text { chegaches / cantaches } \\
\text { (-che- \& -s) }\end{array}$ & 385 & 7 \\
\hline serviu/partiu & 407 & 145 \\
\hline visitaron/cantaron & 350 & 58 \\
\hline foi & 331 & 361 \\
\hline houbo & 373 & 276 \\
\hline puxeron & 356 & 326
\end{tabular}

Tabla 2. Variables analizadas y origen de los datos.

\subsection{P2 de pretérito perfecto (chegaches, cantaches)}

En los datos de los dos proyectos se encuentra información para analizar la variación en la segunda persona de singular de pretérito perfecto. La pregunta 385 del cuaderno I del ALPI (385 ¿A qué hora has llegado?) y los datos del mapa 7 del ALGa (Cantaches) muestran formas de esta variable en dos verbos de la primera conjugación: chegar y cantar. Los materiales de los dos proyectos permiten analizar la variación asociada al morfema de modo y tiempo (-che-) y al morfema de persona y número $(-s)$.

\begin{tabular}{lccc}
\hline FUENTE & -CHE- & -STE- & -CHE- $\&$-STE- \\
\hline$A L P I$ & 50 & 11 & 0 \\
$A L G a$ & 51 & 8 & 2 \\
\hline
\end{tabular}

Tabla 3. Pe de pretérito perfecto (MT).

Las variantes que se registran para el morfema de modo y tiempo son -che-, mayoritaria, y -ste- (Tabla 3). En el ALPI (mapa 1) la segunda variante (chegastes) se anota en una zona compacta del extremo oeste de las provincias de A Coruña y Pontevedra, en un punto aislado del suroeste de Pontevedra y en tres puntos de las provincias de Zamora y León (sureste del dominio). En el mapa del ALGa se observa que la variante mayoritaria se registra como forma única en un punto más y además aparece en competencia con -ste- en dos localidades (mapa 2). La extensión de -che- 
reduce el uso de -ste- como respuesta única y quiebra la continuidad en el área occidental que se descubría en el $A L P I$; la segunda variante se mantiene en tres puntos de A Coruña, dos de Pontevedra y los tres de oriente.

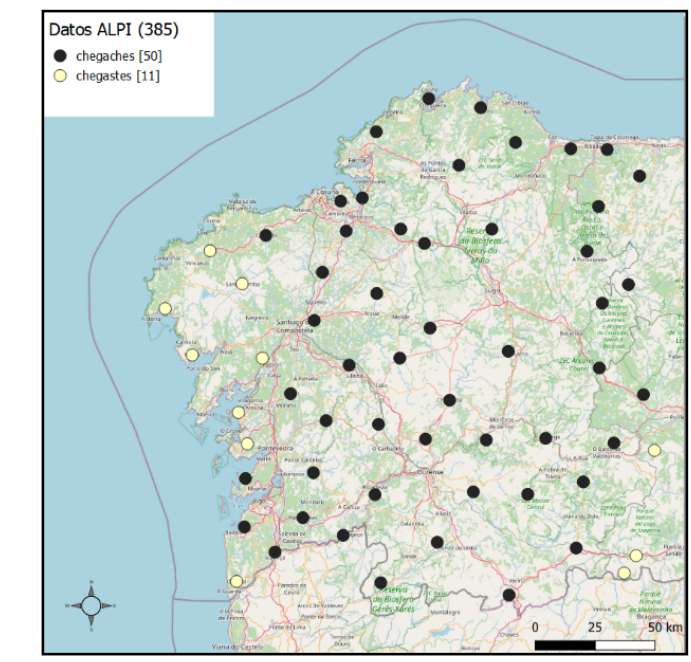

Mapa 1. Chegaches MT $(A L P I)$.

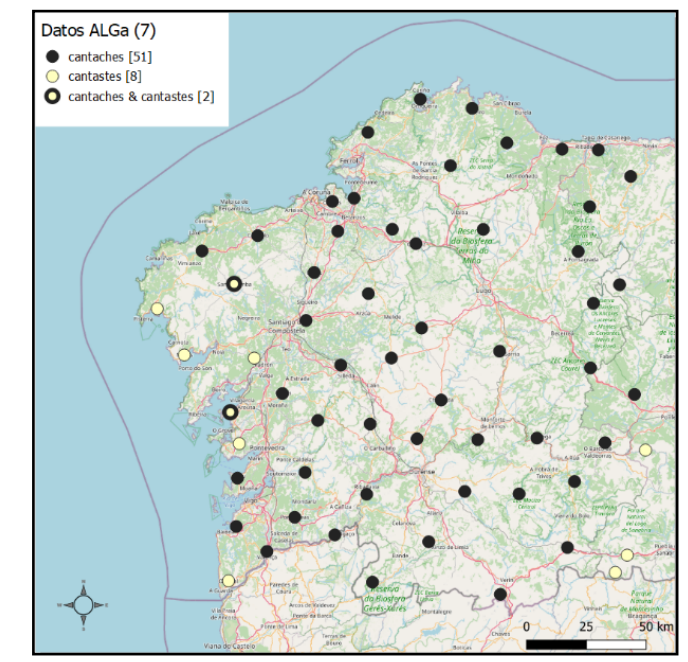

Mapa 2. Cantaches MT (ALGa).

En cuanto a la variación en la expresión del morfema de persona y número, se aprecian diferencias entre los dos proyectos (Tabla 4). Las formas sin -s (cantache y cantaste) son las más comunes en el $A L P I$ y se registran fundamentalmente en el centro y oriente del dominio: provincias de Lugo y Ourense, Rías Baixas de Pontevedra y norte de A Coruña. Las formas con -s (cantaches y cantastes) son más características del tercio occidental: oeste de A Coruña, interior de Pontevedra y puntos aislados de Ourense y Lugo (mapa 3).

\begin{tabular}{lllc}
\hline FUENTE & $-\varnothing$ & $-\varsigma$ & $-\varnothing \&-\varsigma$ \\
\hline$A L P I$ & 41 & 20 & 0 \\
$A L G a$ & 18 & 25 & 18 \\
\hline
\end{tabular}

Tabla 4. P2 de pretérito perfecto: cantaches (NP). 
Los datos del ALGa (mapa 4) muestran una situación diferente, se incrementa el número de registros con -s como respuesta única, disminuyen los casos $\sin -s$ y se anota doble respuesta en muchas localidades del bloque central. Al comparar estos datos con los del $A L P I$, se descubre que las zonas que se muestran como más conservadoras son las situadas en los extremos del dominio: área más oriental y extremo occidental de Pontevedra y A Coruña. Lago (2018) en su trabajo de comparación de los dos proyectos considera más respuestas y muestran que estas diferencias en la aparición de formas con -s también se producen en la segunda conjugación.

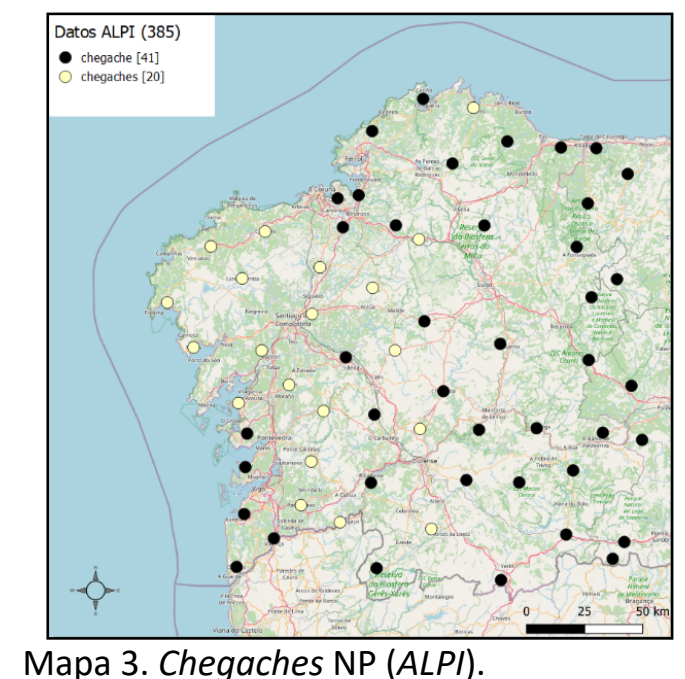

Mapa 3. Chegaches NP (ALPI).

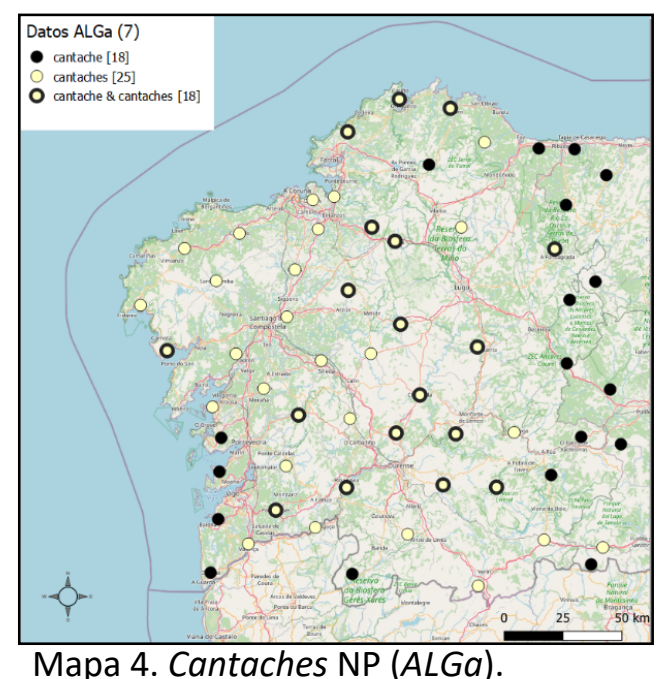

Mapa 4. Cantaches NP $(A L G a)$.

\subsection{P3 de pretérito perfecto (serviu, partiu)}

Las formas de los perfectos arrizotónicos de la tercera conjugación presentan también variación en los materiales de los dos proyectos (Fernández Rei 1990a: 84). Las formas de tercera persona de singular los verbos servir del ALPI (407 Sirvió sólo dos semanas) y partir del ALGa (Mapa 145 Partiu) permiten analizar esta variación (Tabla 5).

Tabla 5. P3 de pretérito perfecto: serviu y partiu.

\begin{tabular}{lcccc}
\hline FUENTE & -EU & $-I U$ & $-I O$ & - EU/-IU \\
\hline$A L P I$ & 17 & 43 & 1 & 0 \\
$A L G a$ & 18 & 42 & 0 & 1 \\
\hline
\end{tabular}

\begin{tabular}{llllll}
$A L G a$ & 18 & 42 & 0 & 1 \\
\hline
\end{tabular} 
En los cuestionarios del $A L P I$ se recogen tres terminaciones para la variable serviu: -iu, -eu y -ío. La variante -iu es la mayoritaria y se registra en todas las provincias, aunque con distinta proporción. Las formas en -eu ocupan una franja diagonal entre el norte de la provincia de Lugo y el norte de la de Pontevedra; -ío se documenta en un punto del este de Lugo. La información cartografiada en el $A L G a$ apenas muestra cambios con respecto al proyecto precedente: no se registra la variante -ío (ahora se anota -iu en el mismo lugar), en zonas de contacto se documentan variantes alternativas y en el punto del municipio de Santiago de Compostela, límite entre las áreas de las dos variantes, se anota una doble respuesta (mapas 5 y 6 ).

Por lo tanto, no se observan mudanzas de relevancia al comparar los datos de los dos proyectos. Las pequeñas diferencias en frecuencia y distribución de las formas indican que hay una situación de relativa estabilidad.

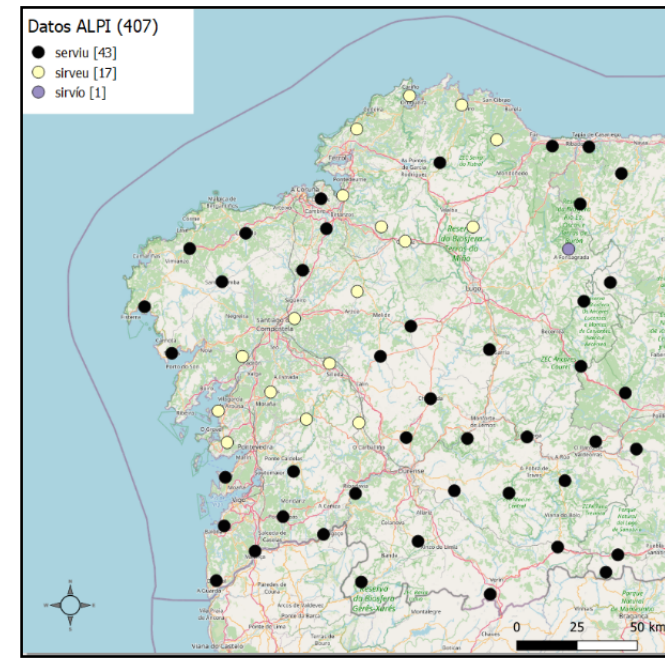

Mapa 5. Serviu $(A L P I)$.

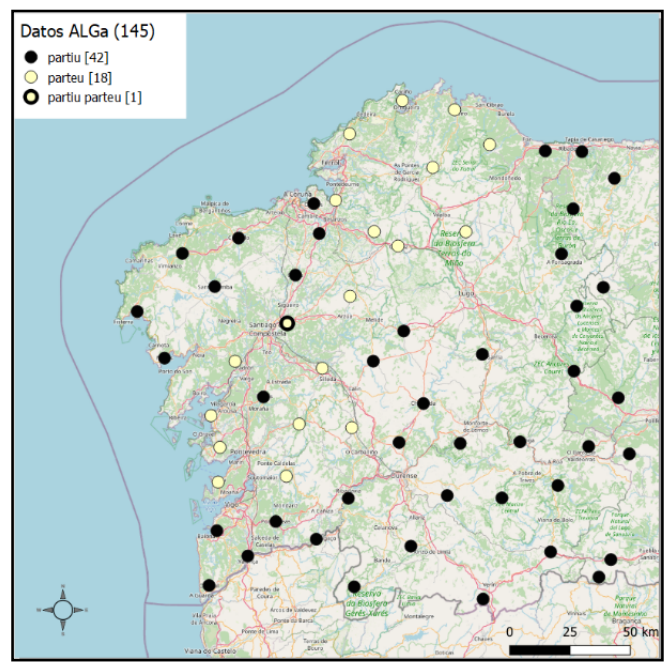

Mapa 6. Partiu (ALGa).

\subsection{P6 de pretérito perfecto (visitaron)}

En el cuestionario del $A L P I$ hay varias preguntas que permiten estudiar en distintos verbos la variación asociada con la tercera persona de plural de pretérito 
perfecto. Nos centraremos en los datos correspondientes a los verbos de la primera conjugación y en los morfemas de modo, tiempo y persona. La pregunta 404 del primer cuaderno del ALPI (350 Visitaron la ciudad) y el mapa 11 del ALGa I (58 Cantaron) ofrecen información sobre esta variable.

\begin{tabular}{lcccc}
\hline FUENTE & -RON & -NON & -NO & -RON/-NO \\
\hline$A L P I$ & 55 & 1 & 3 & 2 \\
$A L G a$ & 56 & 1 & 1 & 3 \\
\hline
\end{tabular}

Tabla 6. P6 de pretérito perfecto: visitaron y cantaron.

Las variantes registradas en el ALPI son -ron, -no y -non (Tabla 6). Visitaron es la variante mayoritaria y se registra por la mayor parte del territorio. La forma visitano se anota en tres localidades occidentales que conforman dos bloques no contiguos (mapa 7) y en otros dos puntos del área como segunda respuesta. Por último, visitanon se recoge en un punto de Pontevedra. En el mapa del ALGa se identifican las mismas variantes, pero en una distribución distinta (mapa 8). La desinencia -ron se registra en la mayor parte del dominio y tanto -no como -non aparecen como variantes en competencia. La comparación de los dos mapas permite suponer que las formas -non y -no debieron ser caracterizadoras de las hablas occidentales, en las que pudieron ocupar un área mayor que la que nos muestran los datos de los dos proyectos. Los cuarenta años transcurridos entre las dos investigaciones supusieron la extensión de la forma más conservadora (-ron), como evidencian los registros de competencia entre formas y de substitución de variantes innovadoras (Fernández Rei 1991: 639). 


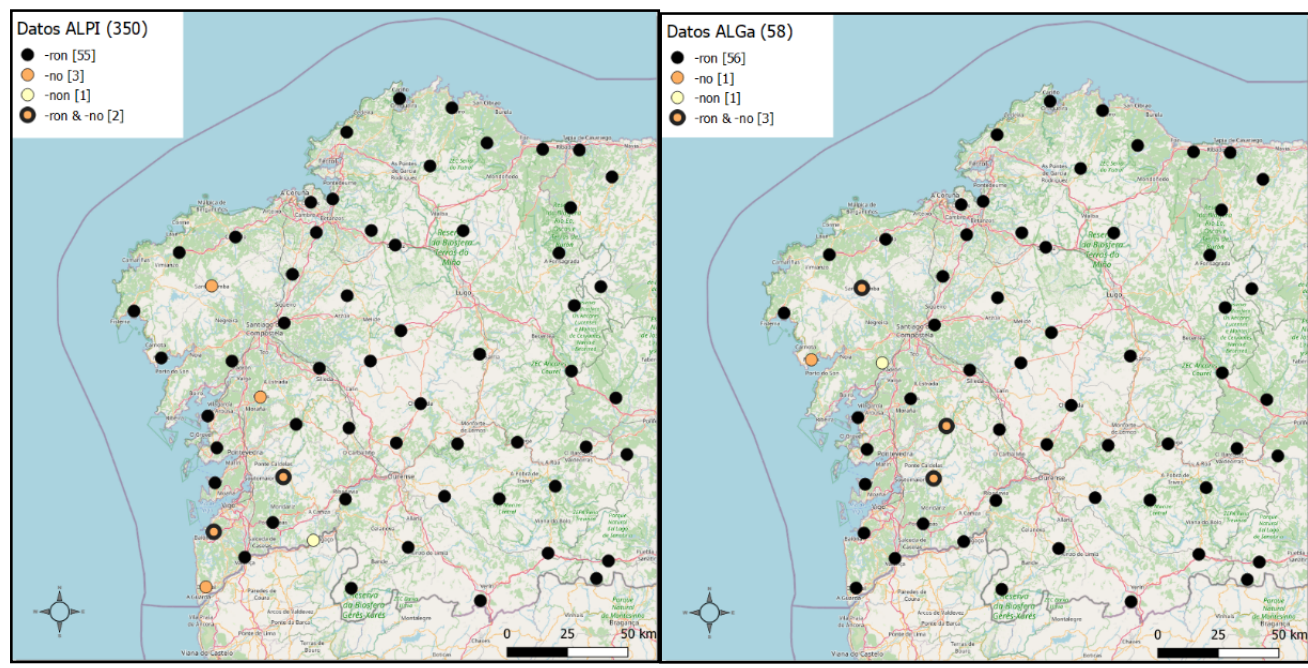

Mapa 7. Visitaron $(A L P I)$.

Mapa 8. Cantaron (ALGa).

\subsection{P3 de pretérito perfecto de ir}

Las variantes registradas para la expresión de la 3a persona de singular de pretérito perfecto de indicativo del verbo ir se tomaron de la pregunta 331 del cuestionario I del ALPI (Fué por avena) y del mapa 361 (Foi) del ALGa. Esta variable no presenta mucha variación. En el ALPI se registraron las variantes foi y fui (Tabla 7); la primera es la forma mayoritaria y la segunda se registró únicamente en el punto 112b, situado en el extremo occidental de la provincia de A Coruña (Mapa 9).

Tabla 7. Tercera persona de pretérito perfecto de ir.

\begin{tabular}{llll}
\hline FUENTE & FOI & FUI & FOI \& FUI \\
\hline$A L P I$ & 60 & 1 & 0 \\
$A L G a$ & 56 & 4 & 1 \\
\hline
\end{tabular}

En la información cartografiada en el $A L G a$ se documentaron las mismas variantes, pero en distinta proporción. La forma foi es la más común y se extiende por todo el dominio, mientras que la variante fui aparece en 4 localidades del occidente de A Coruña, como forma única; además, en una localidad del interior de Pontevedra se anotaron las dos variantes (Mapa 10). 
Si observamos la frecuencia de aparición de las dos variantes y su distribución territorial podemos proponer que la forma fui se extendió a expensas de la otra variante, a pesar de ser forma menos común y divergente. Con respecto a las otras variables analizadas llama la atención que solo se registre un caso de doble respuesta en los datos del ALGa. Por otra parte, como documenta Mariño (2017: 298-299), la alternancia de los diptongos /oi/ / ui/ es antigua y afecta a resultados de distinto origen. En consecuencia, la interpretación de la diferencia observada en los datos debe realizarse con cautela teniendo presente de qué forma pudo influir en las respuestas obtenidas la forma de enunciar la pregunta y la frecuencia de ocurrencia de variación en estos diptongos.

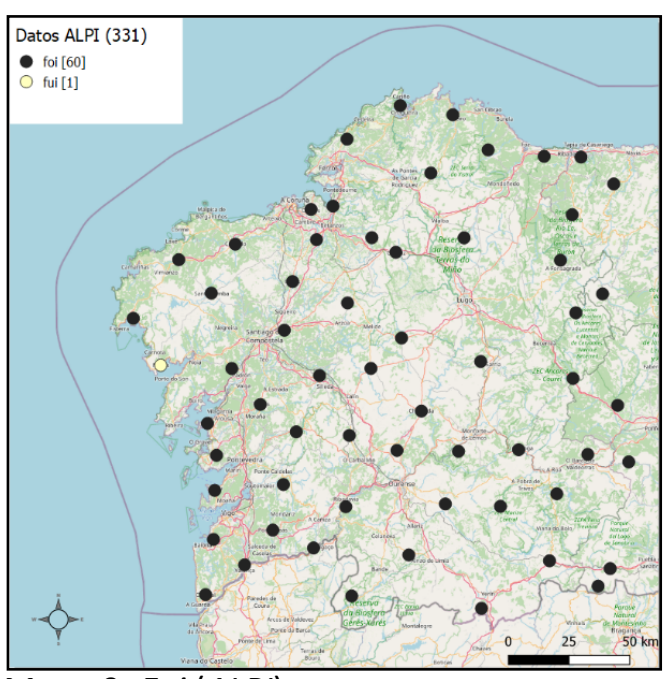

Mapa 9. Foi $(A L P I)$.

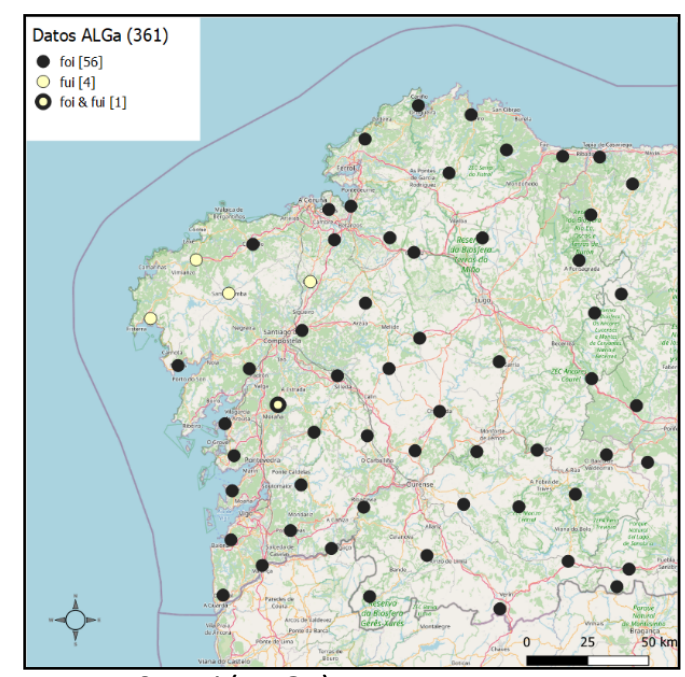

Mapa 10. Foi $(A L G a)$.

\subsection{P3 de pretérito perfecto de haber}

En la pregunta 373 del primer cuaderno del ALPI (EI invierno pasado hubo muchas lluvias) y en el mapa 276 del ALGa (Houben, -eches, -o, -emos, -estes, -eron) se recoge información acerca de la tercera persona de pretérito del verbo haber. Utilizaremos esta información para analizar la variación en el radical de perfecto. Las variantes que figuran en el $A L P I$ son houb-, hub- y hou-, esta última corresponde a la localidad asturiana de As Campas (El inverno pasado hou muitas xeladas). En el ALGa se registran solo las dos primeras (Tabla 8). El radical houb- es el más común y 
extendido en las dos investigaciones. Hub- se registra como forma única en el $A L P I$ en dos puntos del sur de Pontevedra (Mapa 11) y en el ALGa en las mismas localidades, además de en otras tres de Pontevedra y Ourense (Mapa 12). En el ALGa también se anota que en 5 lugares se recogieron respuestas con las dos variantes houb- y hub- en competencia (en 3 puntos del extremo sudoccidental del dominio y en dos del interior de Pontevedra). Los datos muestran un incremento del uso de la forma hub-, que se interpreta como consecuencia del contacto con el español. En el ALPI esta forma aparece restringida a dos localidades, mientras que en el $A L G a$ su uso se extiende a más puntos, desplazando a houb- o bien en competencia con esta y dando cuenta de una situación de variación.

\begin{tabular}{lcccc}
\hline FUENTE & HOUB- & HUB- & HOU- & HOUB- \& HUB- \\
\hline$A L P I$ & 57 & 2 & 1 & 0 \\
$A L G a$ & 51 & 5 & 0 & 5 \\
\hline
\end{tabular}

Tabla 8. Radical de perfecto de haber.

Si se considera la totalidad de las localidades estudiadas en el ALGa (vol I, mapa 276), se puede concluir que la difusión de hub- se produjo de forma rápida y desigual en todo el territorio. Esta expansión está sin duda relacionada con el avance y extensión de uso del español en las décadas que median entre los dos proyectos. En la provincia de A Coruña, en la que no se anotó en el ALPI ningún registro de hub-, se reconoce el uso de esta forma en los años setenta en lugares próximos a núcleos urbanos, como Santiago de Compostela (Mapa 12). 


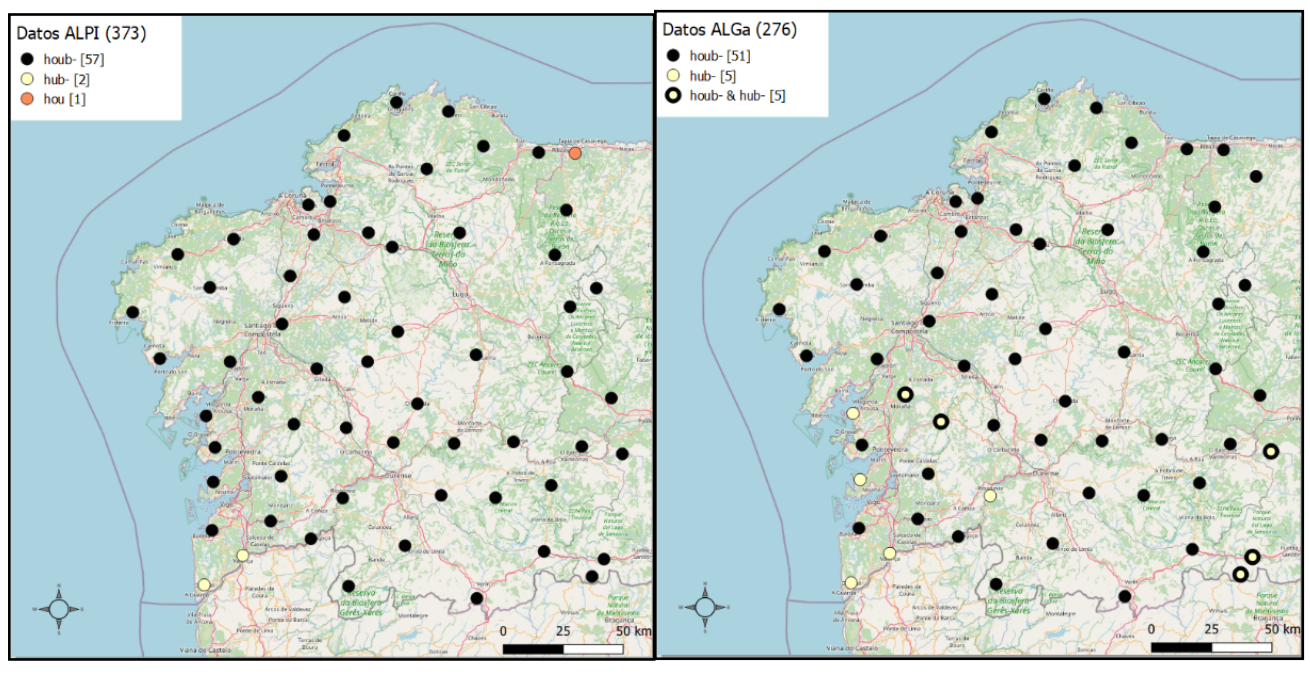

Mapa 11. Houbo (ALPI).

Mapa 12. Houbo (ALGa).

\subsection{6 de pretérito perfecto de poñer}

El análisis de los datos de las dos obras permite conocer la variación y distribución asociada con el radical de perfecto del verbo poñer. Las formas están recogidas en la pregunta 356 del cuestionario I do ALPI (Al niño le pusieron un vestido) y en el mapa 326 del ALGa (Puxemos, -estes, -eron). Para la expresión de esta variable se registraron cuatro variantes: pux-, puñ-, pus- y pue- (Tabla 9). En el ALPI el radical pux- se documenta en la mitad sur de Galicia y occidente de A Coruña. Puñ- aparece registrado en la provincia de Lugo, en el oriente de A Coruña, norte de Ourense y Asturias. Los cuatro registros de pus- corresponden a 3 puntos próximos situados en el extremo sudoriental de dominio (Ourense y Zamora) y a una localidad del sur de Ourense; las cuatro localidades están cercanas a la frontera portuguesa. La variante pue- (pueron) se registró en una localidad de Asturias. En localidades próximas a los límites entre las áreas de pux- y puñ-se registran dobles respuestas (Mapa 13).

En el $A L G a$ pux- aparece en un área compacta que ocupa todo el territorio excepto una parte de la provincia de Lugo, en la que la única variante registrada es puñ- (mapa 14). Entre estas dos zonas aparecen documentados un número importante de puntos intermedios con doble respuesta (pux- y puñ-). También se anotó puñ- en localidades aisladas del sur del sur de Pontevedra, Asturias y León. El radical pus- 
aparece en el externo occidental de A Coruña y en tres puntos orientales (León y Zamora).

\begin{tabular}{lcccccc}
\hline FUENTE & PUX- & PUÑ- & PUS- & PUE- & PUX- \& PUÑ- & PUX- \& PUS- \\
\hline$A L P I$ & 30 & 21 & 4 & 1 & 5 & 0 \\
$A L G a$ & 24 & 18 & 6 & 1 & 10 & 2 \\
\hline
\end{tabular}

Tabla 9. Radical de perfecto de poñer.

Las variantes mayoritarias (pux- e puñ-) coinciden en los dos proyectos con una diferencia que puede asociarse con la tendencia en el $A L G a$ a tomar registro de las dobles respuestas. Las diferencias se aprecian más en la distribución territorial de las formas y en las segundas respuestas anotadas. Llaman la atención las localidades que en el $A L P I$ mostraban una única respuesta y en el $A L G a$ aparecen con doble repuesta, y también las localidades que aparecían con doble respuesta ya en el $A L P I$ y que en el ALGa figuran también como doble respuesta, pero siempre con pux- anotado en primer lugar. Además, se aprecian puntos en los que variante puñ- del ALPI es desplazada por pux-. Estos dos hechos parecen apuntar a un cambio que favorece la expansión del radical mayoritario pux- (vid. Dubert 2014, 2017). Los datos abonan otra vez como la variación (dobles respuestas) documentada en el ALPI da lugar a cambios registrados en el proyecto desarrollado cuatro décadas más tarde (de dobles respuestas a respuestas únicas).

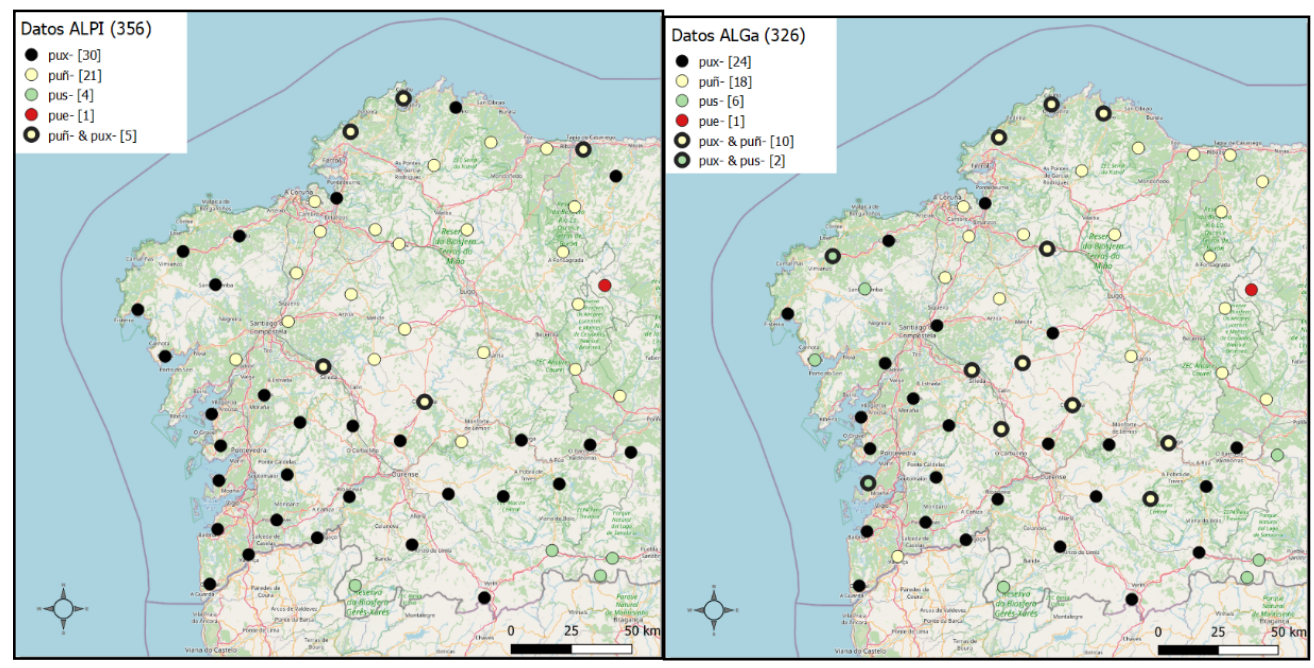

Mapa 13. Pux- $(A L P I)$.

Mapa. 14 Pux- (ALGa). 
La distribución del radical pus- se muestra con poca variación en el extremo suroccidental, pero se documenta en dos localidades para las que en el ALPI se anotó pux-. El registro de esta forma debe ponerse en relación con el fenómeno identificado como despalatalización de la consonante fricativa lámino-postalveolar / / común a variedades de esta área (Fernández Rei 1990b).

\section{Conclusiones}

Los estudios de cambio lingüístico en tiempo real pueden recurrir a comparar los datos de dos fuentes documentales distanciadas en el tiempo o bien a encuestar a una comunidad en el presente y contrastar el resultado con documentación pasada (CukorAvila \& Bailey 2013). Los atlas lingüísticos deben ser utilizados como fuentes documentales que proporcionan información de valor extraordinario para reconstruir la historia y evolución de las variedades lingüísticas. Para el conjunto de dialectos del dominio iberorromance el $A L P I$ constituye un tesoro de especial valor para indagar los cambios que en los distintos niveles de los sistemas lingüísticos se fueron produciendo en la península desde el primer tercio del siglo pasado.

El propósito de este trabajo ha sido demostrar la utilidad de los materiales de los atlas lingüísticos para el estudio de los cambios en la morfología verbal. Los resultados indican que en las variables morfológicas analizadas no se observan cambios profundos. El número de variantes y su distribución geográfica, de forma general, no muestran muchas mudanzas. Con todo, se pueden señalar algunas diferencias de interés. En primer lugar, destaca el incremento de los casos de variación en el ALGa: localidades para las que en el $A L P I$ se registraba una única respuesta aparecen en el ALGa con dos respuestas en competencia (cantaches y cantache, -ron y -non, houb- y hub-, pux- y puñ-); esta tendencia ya se había indicado en trabajos anteriores sobre variables fonéticas y léxicas (Sousa 2012; Sousa \& Dubert 2020). Esta diferencia permite proponer que las variedades dialectales identificadas en el ALPI se muestran más estables, con menor variación, que las estudiadas en el ALGa. Por otra parte, se 
aprecia que las localidades más permeables a los cambios (cambio de variante o incremento de la variación) se sitúan en el área occidental del territorio, la zona más poblaba y con más núcleos urbanos. En último término, la confrontación de estos resultados con estudios previos sobre los mismos materiales parece indicar que las variables morfológicas analizadas manifiestan un comportamiento similar al de las variables fonológicas, con cambios escasos y de poca entidad (Sousa 2010), y diferente del que se aprecia en el nivel léxico (Sousa \& Dubert 2020). Con todo, como antes se ha señalado, los resultados de este trabajo son limitados y será necesario un estudio de mayor profundidad y con más datos para comprobar la validez de nuestras hipótesis.

En el ámbito de los estudios lingüísticos se tiene la idea de que la contribución de los proyectos de geografía lingüística se culmina con la publicación de los atlas. Esta creencia tiene cierto fundamento, ya que los materiales registrados para los atlas lingüísticos pocas veces son aprovechados para análisis fuera del grupo de investigadores que participan en los proyectos. Una de las razones que explica esta situación es la dificultad que supone manejar y extraer la información albergada en los volúmenes de un atlas lingüístico publicado en papel. La tecnología informática permite hoy que los datos sean accesibles para la comunidad científica en formatos reutilizables y fáciles de consultar. Esta nueva forma de acceso y difusión de la información geolingüística puede ser aplicada tanto a los nuevos proyectos como a proyectos finalizados que de esta manera cobran una nueva vida. Un ejemplo paradigmático de este renacimiento es el atlas de Wenker, considerado el primer gran atlas lingüístico europeo. En la actualidad los materiales originales del proyecto están accesibles de forma simple a través de una página web (Glaser 2020) y además han dado lugar a un proyecto interesante de ciencia ciudadana que busca comparar los datos recogidos en el siglo XIX con las variedades del alemán actual. En el entorno de la empresa de reedición de los materiales del ALPI también han surgido varios proyectos de investigación que permiten imaginar un futuro fructífero a la obra ideada por Menéndez Pidal y Navarro Tomás hace un siglo. 


\section{Referencias}

ÁlVAREZ, Rosario, Francisco DUbert \& Xulio SOUSA (2020) Atlas Lingüístico Galego: Volume VII. Léxico. O ser humano (II), Santiago de Compostela: Fundacion Barrié; Universidade de Santiago de Compostela.

BRITAIN, David (2014) “Geographical dialectology", en Janet Holmes \& Kirk Hazen (eds.), Research Methods in Sociolinguistics: A Practical Guide, Hichester, UK: Wiley Blackwell, 246-261.

Cortés Carreres, Santi \& Vicent García Perales (eds.) (2009), La historia interna del Atlas Lingüístico de la Península Ibérica (ALPI). Correspondencia (1910-1976), Valencia: Universitat de València.

CUGNo, Federica \& Lorenzo MASSOBRIO (2014) Gli atlanti linguistici della Romània. Corso di geografia lingüística, Alessandria: Edizioni dell'Orso.

CUKOR-AVILA, Patricia \& Guy BAILEY (2013) "Real time and apparent time", en J. K. Chambers \& Natalie Schilling (eds.), The Handbook of Language Variation and Change, 2nd Edition, Oxford: Wiley, 239-262.

DALBERA, Jean-Philippe (2007) "Linguistic Atlases. Objectives, Methods, Results, Prospects", en Yuji Kawaguchi, Toshihiro Takagaki, Nobuo Tomimori \& Yoichiro Tsuruga (eds.), Corpusbased perspectives in linguistics, Amsterdam, Philadelphia: John Benjamins, 39-54.

DUbert García, Francisco (2017) “Cambio e estrutura morfolóxica. Segmentacións atípicas en verbos galegos e portugueses", en Xosé Luís Regueira \& Elisa Fernández Rei (eds.), Estudos sobre o cambio lingüístico no galego actual, Santiago de Compostela: Consello da Cultura Galega, 297-326.

DUBerT, Francisco (2014) "Splinter morphologique et analogie dans les dialectes galiciens et portugais", en Morphologie flexionnelle et dialectologie romane : typologie(s) et modélisation(s), Mémoires de la Société de Linguistique de Paris 22, Louvain: Peeters, 69-87.

FERnÁNDEZ Rel, Francisco (1990a) Atlas Lingüístico Galego. Volume I. Morfoloxía verbal, A Coruña: Fundación Pedro Barrié de la Maza.

FERNÁNDEZ REI, Francisco (1990b) Dialectoloxía da lingua galega, Vigo: Xerais. 
FERNÁNDEZ REI, Francisco (1991) “Conservadurismo e innovación no sistema de desinencias verbais do galego: o perfecto", en D. Kremer (ed.), Actes du XVIIle Congrès International de Linguistique et de Philologie Romanes, Tübingen: Niemeyer, 632-644.

FERNÁNDEZ REI, Francisco (1993) “Atlas lingüístico Galego: I. Morfoloxía verbal”, en R. Lorenzo (ed.), Actas do XIX Congreso Internacional de Lingüística e Filoloxía Románicas, Santiago de Compostela: Fundación Pedro Barrié de la Maza, 617-637.

FERNÁNDEZ REI, Francisco (2007) "A contribución de Alonso Zamora Vicente", Revista de Filología Románica, 24, 13-27.

García Mouton, Pilar (2014) Lenguas y dialectos de España, Madrid: Arco Libros.

García Mouton, Pilar (coord.), Inés Fernández-Ordóñez, David HeAP, Maria Pilar PereA, João SARAMAGO \& Xulio SOUSA (2016) ALPI-CSIC [www.ALPI.csic.es], edición digital de Navarro Tomás, Tomás (dir.), Atlas Lingüístico de la Península Ibérica, Madrid: CSIC.

García, Constantino, Antón Santamarina, Rosario Álvarez Blanco, Francisco Fernández Rei \& Manuel GonZÁLEZ GonZÁLEZ (1977) “O Atlas Lingüístico Galego”, Verba, 4, 5-17.

GIMENO MENÉNDEZ, Francisco (1990) Dialectología y sociolingüística españolas, Alicante: Universidad de Alicante.

GLASER, Elvira 2020 Projekt Wenker. Zürich: Universität Zürich <https://wenker.citizenscience.ch/de/>.

GonZÁLEZ GonZÁLEZ, Manuel (1991) “Metodología de los Atlas lingüísticos en España”, IKER 7, 151-177.

KEMP, J. Alan (2006) "Phonetic transcription: History", en Keith Brown (ed.), The encyclopedia of language \& linguistics, 2nd ed, Vol. 9, Amsterdam: Elsevier, 396-410.

LAGo CAAMAÑo, Clara (2018) Cambio lingüístico en galego no século XX: os pretéritos verbais. Trabajo de Fin de Grado, Santiago de Compostela: Universidade de Santiago de Compostela.

MARIÑO PAZ, Ramón (2017) Fonética e fonoloxía históricas da lingua galega, Vigo: Xerais.

MENÉNDEZ PIDAL, Ramón (1926) Orígenes del español: estado lingüístico de la Península ibérica hasta el siglo XI, Madrid: Librería y Casa Editorial Hernando.

NAVARro Tomás, Tomás (1975) "Noticia histórica del ALPl", en Tomás Navarro Tomas (ed.), Capítulos de geografía lingüística de la Península Ibérica, Bogotá: Instituto Caro y Cuervo, 9-20. 
NAVARRo Tomás, Tomás (dir.), Francesc de BorJA Moll, Aurelio M. EsPINOSA [hijo], Luís F. LINDLEY Cintra, Armando Nobre de Gusmão, Aníbal Otero, Lorenzo Rodríguez Castellano \& Manuel SAnchis Guarner (1962) Atlas lingüístico de la Península Ibérica, vol. 1, Fonética, Madrid: CSIC.

SOUSA FERNÁNDEZ, Xulio (2010) "Xeolingüística e cambio lingüístico: gheada e seseo no ALPI e no $A L G a^{\prime \prime}$, en Maria Iliescu, Heidi Siller-Runggaldier \& Paul Danler (eds.), Actes du XXVe Congrès International de Linguistique et de Philologie Romanes, Berlín: De Gruyter, 257-267.

SOUSA FERNÁNDEZ, Xulio (2012) "Dialect change and variation: the Atlas Lingüístico de la Península Ibérica", Dialectologia, 3, 189-207.

$<$ http://www.publicacions.ub.edu/revistes/dialectologia3/>

SOUSA, Xulio (en prensa) "El Atlas Lingüístico de la Península Ibérica y el gallego del siglo XX", en Pilar García Mouton \& Isabel Molina Martos (eds.), Investigaciones geolingüísticas en la Península Ibérica, Madrid: Revista de Filología Española.

SOUSA, Xulio \& David RodríGUez (2017) "Do espazo ó tempo: o cambio lingüístico en galego a partir da xeolingüística", en X. L. Regueira \& Elisa Fernández Rei (eds.), Estudos sobre o cambio lingüístico no galego actual, Santiago de Compostela: Consello da Cultura Galega, 243-269.

SOUSA, Xulio \& Francisco DUBeRT-GarCíA (2020) “Measuring language contact in geographical space: Spanish loanwords in Galician", Zeitschrift für Dialektologie und Linguistik 87(2), 285-306. doi.org/10.25162/zdl-2020-0010

VIDOS, Benedek Elemér (1973) Manual de lingüística románica, Madrid: Editorial Gredos.

\section{Anexo 1}

Correspondencia entre los puntos de los dos proyectos analizados. Los códigos corresponden a la forma de identificación de las localidades en cada uno de los atlas. En García Mouton et al. (2016) y en Álvarez, Dubert y Sousa (2020) se pueden consultar relaciones detalladas de las localidades. 
Dialectologia. Special issue, IX (2021), 285-310.

\begin{tabular}{|c|c|}
\hline Punto ALPI & Punto $A L G a$ \\
\hline 100 & C.03 \\
\hline 101 & C.04 \\
\hline 102 & C.09 \\
\hline 103 & C.10 \\
\hline 104 & C.19 \\
\hline 105 & C.20 \\
\hline 106 & C. 22 \\
\hline 107 & C.18 \\
\hline 108 & C.34 \\
\hline 109 & C. 27 \\
\hline 110 & C.31 \\
\hline 111 & C.26 \\
\hline 112 & C.35 \\
\hline $112 a$ & C.43 \\
\hline 113 & C.46 \\
\hline 114 & L.01 \\
\hline 115 & L.07 \\
\hline 116 & L.05 \\
\hline 117 & L.04 \\
\hline 118 & L.17 \\
\hline
\end{tabular}

\begin{tabular}{|c|c|}
\hline 119 & L.14 \\
\hline 120 & L.16 \\
\hline 121 & L. 28 \\
\hline 122 & L. 23 \\
\hline 123 & L. 26 \\
\hline 124 & L. 29 \\
\hline 125 & L.36 \\
\hline 126 & L.39 \\
\hline 127 & L.38 \\
\hline 128 & P.02 \\
\hline 129 & P.05 \\
\hline 130 & P.09 \\
\hline 131 & P.12 \\
\hline 132 & P.16 \\
\hline 133 & P.14 \\
\hline 134 & P.18 \\
\hline 135 & P.17 \\
\hline 136 & P.28 \\
\hline 137 & P.25 \\
\hline 138 & P.27 \\
\hline 139 & P.29 \\
\hline
\end{tabular}

\begin{tabular}{|c|c|}
\hline 140 & P.33 \\
\hline 141 & 0.02 \\
\hline 142 & 0.01 \\
\hline 143 & 0.15 \\
\hline 144 & 0.14 \\
\hline 145 & 0.13 \\
\hline 146 & 0.12 \\
\hline 147 & 0.03 \\
\hline 148 & 0.20 \\
\hline 149 & 0.21 \\
\hline 150 & 0.26 \\
\hline 151 & 0.30 \\
\hline 300 & A. 5 \\
\hline 301 & A.1 \\
\hline 303 & A. 4 \\
\hline 323 & A.7 \\
\hline 324 & Le. 2 \\
\hline 333 & Le.5 \\
\hline 340 & Z.2 \\
\hline 341 & Z.3 \\
\hline
\end{tabular}




\section{Anexo 2}

Mapa de la red de puntos de los dos proyectos estudiados. Los números de las etiquetas corresponden a la codificación utilizada en al $A L P I$.

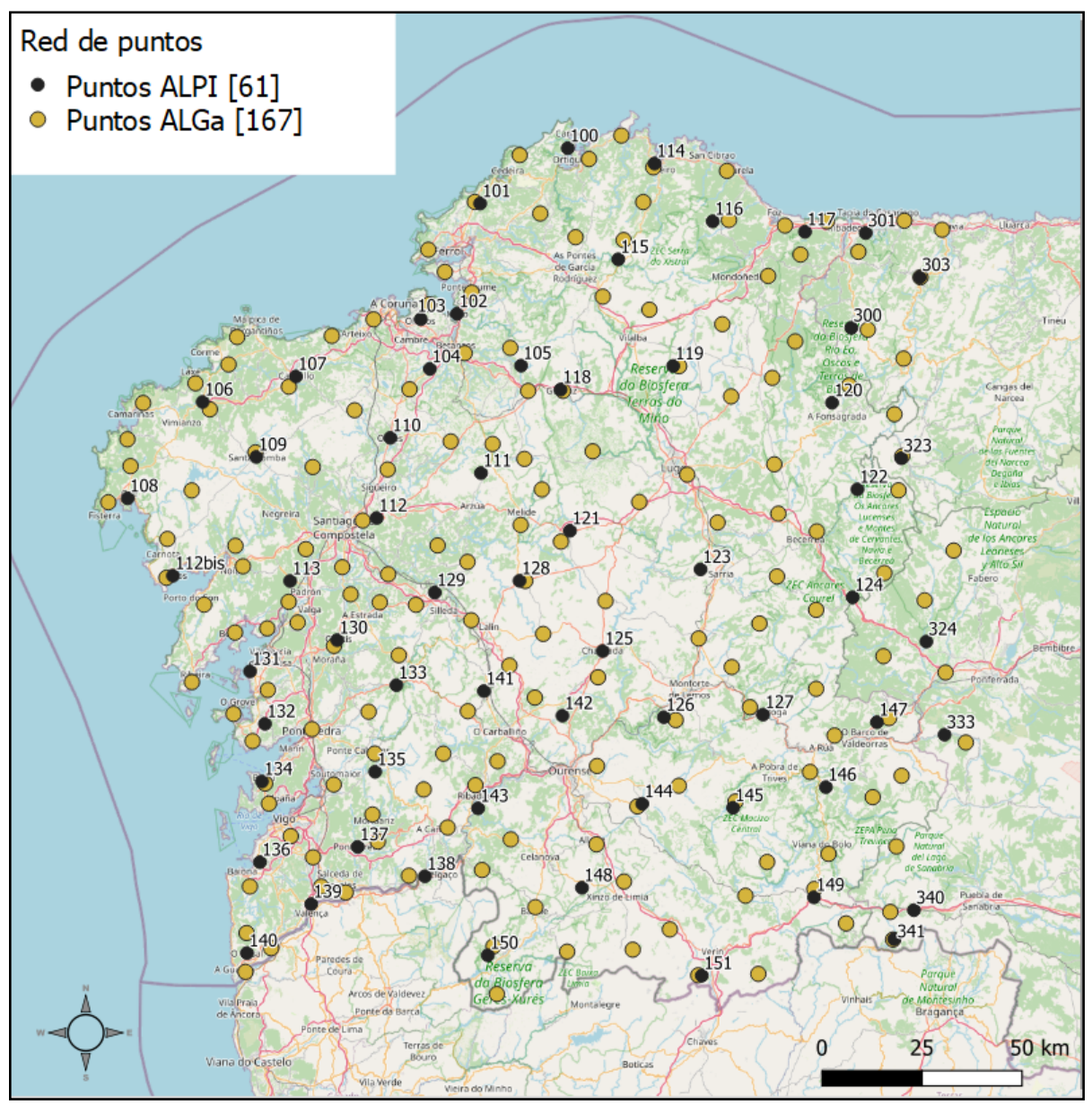

